\title{
Prevalence of Candida Species among HIV Positive Patients in Two Tertiary Hospitals in Rivers State
}

\author{
Ndukwu, C. B ${ }^{1}$, C. Mbakwem-Aniebo ${ }^{2}$, N. Frank-Peterside ${ }^{2}$ \\ ${ }^{l}$ Department of Medical Laboratory Science, Medical Microbiology Unit, Braithwaite Memorial Specialist \\ Hospital, Port Harcourt, Rivers State \\ ${ }^{2}$ Department of Microbiology, University of Port Harcourt, Nigeria.
}

\begin{abstract}
The study investigated the prevalence of Candida species among HIV positive patients in two tertiary hospitals in Rivers State. A total of 300 samples of sputum, throat swab and high vaginal swab samples were obtained from HIV positive patients attending Braithwaite Memorial Specialist Hospital (BMSH) and the University of Port Harcourt Teaching Hospital (UPTH). The Candida species identified from the samples include; C. albicans, C. stellatoidea and C. tropicalis. The mean distribution of C. albicans, C. stellatoidea and C. tropicalis among the study subjects were $179.00 \pm 11.36,7.00 \pm 1.00$ and $72.33 \pm 27.10$ respectively. Sputum samples obtained from HIV positive patients had the highest prevalence of Candida species (56.3\%), followed by throat swab sample (30.7\%) and high vaginal swab samples (13.0 \%). Prevalence of candidiasis among HIV patients was revealed to be higher among subjects between the ages of $26-33$ years followed by 18 - 25 years, $34-41$ years, $42-49$ years, 50 - 57 years and $58-65$ years. The compromised immune systems of HIV positive patients permits the multiplication of Candida species leading to infections.
\end{abstract}

\section{Introduction}

Globally, an estimated 35.3 million people were living with HIV, 2.3 million people became infected, and 1.6 million deaths occurred in the year 2012 (UNAIDS, 2013). Owing to a weakened immune e system, the infected person is placed at an increased risk of a wide variety of opportunistic infections (Shahapur and Bidri, 2014). Fungal opportunistic infections such as candidiasis in patients infected with HIV are a major cause of morbidity and mortality and compromise the quality of life of such individuals (Durden and Elewsi, 1997). The spectrum of Candida infection is diverse starting from asymptomatic colonization to oropharyngeal candidiasis, esophagitis, onychomycosis, vulovaginitis, cutaneous candidiasis and systemic candidiasis or invasive candidiasis including candedemia. Candidiasis is attributed to a reduction in host immune defences. A change in distribution profile of Candida specie can be an indication of drug resistance or immunosuppression levels in populations. It could be a sensitive and specific indicator of a decrease in the number of CD4 cells and would show the onset of significant immune deficiency in people with HIV. The first step in the development of a Candida infection is colonization of the mucocutaneous surfaces. The most common agent of candidiasis are Candida albicans, C. glabrata, C. krusei, C. tropicalis, C. parapsilosis, C. dubliniensis and C. gulliermondi. (Sant'Ana et al., 2002). Non albican species are implicated with greater frequency as opportunistic pathogens associated with diseases especially host (Baradkar and Kumar, 2008). The different species of Candida that causes candidiasis in HIV patients if not identified and properly treated with the appropriate drug could lead to resistance of the drug and make treatment very difficult.

\section{Sample Collection}

\section{Materials And Method}

High vaginal swabs (HVS) and throat swabs were collected from patients attending Braithwaite Memorial Specialist Hospital (BMSH) and the University of Port Harcourt Teaching Hospital (UPTH) using sterile swab sticks while the early morning sputum of the confirmed tuberculosis subjects were collected for wet prep and culture. Samples were collected in pairs.

\section{Microscopy}

The first set of swab sticks (HVS and Throat swabs) were agitated in approximately $1 \mathrm{ml}$ of normal saline put in different test tubes. A drop of suspension of each sample was transferred to a different grease free microscope slide. Cover slip was placed gently to exclude air bubbles and viewed microscopically under 10x and 40x objectives (Al-Aali, 2010). Sputum samples were placed on clean grease-free slides and a drop of potassium hydroxide $(\mathrm{KOH})$ added. The preparation were mixed and covered with a cover glass. This was examined for fungi using 10x and 40x objectives. 
Prevalence Of Candida Species Among Hiv Positive Patients In Two Tertiary Hospitals In Rivers...

\section{Cultivation}

The second set of swab sticks (HVS and Throat swabs) were plated out by streaking on modified Sabouraud-chloramphenical agar plates and incubated at $37^{\circ} \mathrm{C}$ for up to 72 hours (Al-Aali, 2010). The antibiotics $0.5 \%$ chloramphenicol, inhibited bacterial growth. On observation, the positive plates had entire edges, cream coloured colonies with pasty smell that is typical of Candida spp.

\section{Biochemical tests}

These tests were carried out according to the method of CDC (2010). Candida spp. were differentiated from other yeasts and were identified to specie level using Gram stain, morphology, germ tube formation, corn meal agar with tween-80(for demonstration of chlamydospores, blastospores and pseudohypae) sugar fermentation test(glucose, sucrose lactose,maltose and xylose) closing as confirmatory test. Gram staining was done from suspected yeast colonies only those with budding yeast cells and pseudohypae along with pus cell spectrum and heavy growth of Candida with more than 30 colonies on SDA were considered this is to exclude normal flora. Formation of germ tube at $37^{\circ} \mathrm{C}$ in horse serum after $2 \mathrm{hrs}$ indicated a positive result. Sugar assimilation /fermentations reaction were carried out by incorporating $2 \%$ of various sugars (maltose, sucrose, lactose, xylose and glucose) into broth medium with indicators in sterile narrow neck MaCartney bottles containing Durham tubes. Tubes were incubated at $37^{\circ} \mathrm{C}$ overnight.

\section{Results}

The prevalence of candidiasis among HIV subjects in two tertiary hospitals in Rivers State is shown in Table 1. BMSH recorded 290 HIV patients with candidiasis co-infection out of $481 \mathrm{HIV}$ patients examined while $240 \mathrm{HIV}$ patients with candidiasis co-infection was recorded in UPTH out of a total of 300 HIV patients examined. The number of HIV patients without candididiasis in BMSH and UPTH were 191 and 69 respectively. The mean distribution of phenotypically characterized Candida species among HIV patients is shown in Table 2. The mean distribution of $C$. albicans, $C$. stellatoidea and $C$. tropicalis among the study subjects were $179.00 \pm 11.36,7.00 \pm 1.00$ and $72.33 \pm 27.10$ respectively. The percentage distribution of Candida spp. by site is shown in Table 3. The highest distribution of candidiasis was recorded in sputum samples $(56.3 \%)$, followed by throat swab sample $(30.7 \%)$ and high vaginal swab samples $(13.0 \%)$. Prevalence of candidiasis among different age groups is shown in Figure 1. Results revealed candidiasis was predominant among the age bracket of $26-33$ years with a total of 80 co-infected patients out of 300 . This was followed by patients in the age bracket of $18-25$ years with 66 co-infected patients. The age group with the least candidiasis distribution was 58 - 65 years, 30 co-infected patients were recorded in this group.

Table 1: Prevalence of Candidiasis in the Study Area

\begin{tabular}{|l|l|l|l|}
\hline Study Area & No. of Adults Examined & No. of Adults Infected & No. of Adults Uninfected \\
\hline BMSH & 481 & 290 & 191 \\
\hline UPTH & 300 & 240 & 69 \\
\hline
\end{tabular}

Table 2: Mean Distribution of Phenotypically Characterized Candida species among HIV patients

\begin{tabular}{|l|l|}
\hline Isolate & Mean Distribution \\
\hline Candida albicans & $179.00 \pm 11.36^{\mathrm{a}}$ \\
\hline C. stellatoidea & $7.00 \pm 1.00^{\mathrm{c}}$ \\
\hline C. tropicalis & $72.33 \pm 27.10^{\mathrm{b}}$ \\
\hline
\end{tabular}

a,b,c: Means with different superscript are statistically significantly $(\mathrm{P}>0.05)$ different

Table 3: Percentage Distribution of Candida species by Site Among HIV Patients

\begin{tabular}{|l|l|}
\hline Isolate & Percentage Distribution \\
\hline High Vaginal Swab & 13.0 \\
\hline Sputum & 56.3 \\
\hline Throat & 30.7 \\
\hline
\end{tabular}

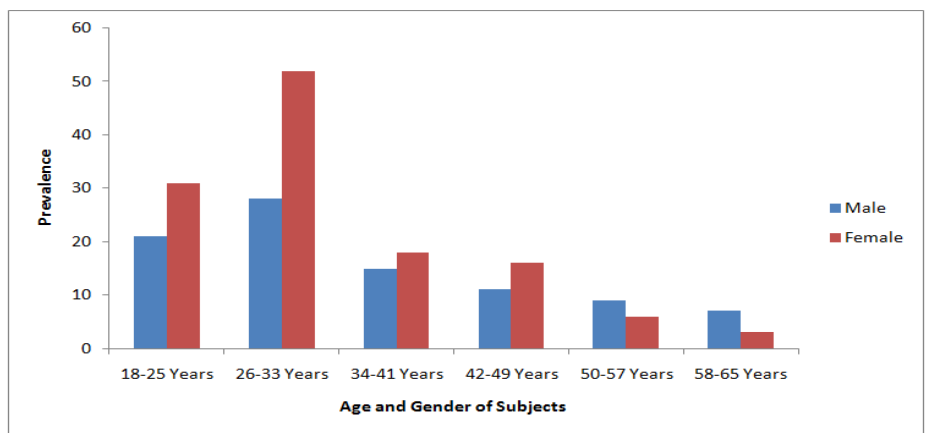

Figure 1: Prevalence of Candidiasis among different age groups 


\section{Discussion}

The mean distribution of phenotypically characterized Candida spp among HIV patients revealed $C$. albicans was the highest occurring Candida sp followed by $C$. tropicalis and $C$. stallatoidea. The mean distribution of the 3 isolates were $179.00 \pm 11.36,7.00 \pm 1.00$ and $72.33 \pm 27.10$ for C. albicans, $C$. stellatoidea and $C$. tropicalis respectively. Statistical analysis carried out using one way Anova revealed that mean distribution of all isolates were statistically significant $(\mathrm{P}<0.05)$. In a similar study carried out by Thanyasrisuy et al. (2014), C. albicans was the predominant specie among all Candida isolates. In another study C. albicans (50\%) was isolated in $60 \%$ of samples obtained from HIV patients further suggesting that C. albicans is the major Candida specie commonly implicated in candidiasis among HIV patients. The other Candida isolates include C. tropicalis (20\%), C. parapsilosis (19.3\%), C. guillerimondi (4.8\%) and C. krusei (1.6\%) (Costa et al., 2008). Candida albicans is an opportunistic pathogen. Immunosuppression and the indiscriminate use of antimicrobial agents apparently allows its multiplication and colonization of the oropharynx leading to diseases ranging from superficial to systemic infections in both children and adults (Enwurul et al., 2008). Isolation of $C$. tropicalis among HIV patients have also been reported (Maheshuwari et al., 2016; Thanyasrisury et al., 2014). However, reports on the isolation of $C$. stellatoidea from HIV subjects are few suggesting that the isolate is not a major cause of candidiasis among HIV patients. Percentage distribution of Candida spp. by site revealed a higher distribution in sputum and throat swab samples with percentage distribution of $56.3 \%$ and $30.7 \%$ respectively. The least percentage distribution was recorded from high vaginal swab (HVS) with $13.0 \%$. In a similar study carried out by Anwar et al. (2012), 68 Candida species were obtained from oral swab out of a total of 94 Candida species, 12 species were obtained from the skin, 6 species from stool, 3 species from blood, 3 species from sputum and 2 species from oesophageal biopay. Candida species may be localized in the mouth, lungs or the gastrointestinal tract of HIV patients (Maheshuwari et al. 2016). It has been observed that low CD4 counts and high plasma HIV RNA levels significantly correlate with oral Candida carriage as well as with oral candidiasis in HIV patients (Liu et al. 2006). Prevalence of candidiasis among HIV patients was revealed to be higher among subjects between the ages of $26-33$ years followed by $18-25$ years, $34-41$ years, $42-49$ years, $50-57$ years and $58-65$ years. Though a retrospective study to determine the use of antiretroviral drugs among the subjects was not carried out, very high prevalence of candidiasis among HIV patients between 26 33 years of age suggests poor management of the virus leading to a more compromised immune system. The result also suggests carelessness in sexual activities among this age group given that this age group consists mainly of sexually active persons.

\section{Conclusion}

The study has shown that candidiasis is prevalent among HIV subjects and this is largely due to a compromised immune system as a result of the viral activities in the host cell. There is need therefore to routinely check for opportunistic infections especially in the case of an immunocompromised individual. This will help to monitor disease progression and complications.

\section{Reference}

[1]. Al-Aali, K.Y. (2013). Prevalence of Vaginal Candidiasis among Pregnant Women Attending Al-Hada Military Hospital, Western Region, Taif, Saudi Arabia. International Journal of Science and Research. 4 (5): 1736 - 1743.

[2]. Anwar, K.P., Malik, A. and Subhan K.H. (2012). Profile of Candidiasis in HIV Infected Patients. Iranian Journal of Microbiology. 4 (4): $204-209$.

[3]. Calderone R.A. (2001). Candida and candidiasis. Washington DC: ASM Press.

[4]. Costa, C.R.., Cohen, A.J., Fernandes O.F.L., Miranda, K.C., Passos, K.S. Souza A.K.H. and Silva R.R. (2016). Asymptomatic Oral Carriage of Candida species in HIV-infected Patients in the Highly Active Antiretroviral Therapy Era. Rev. Inst. Med. Trop. 48 : $257-261$.

[5]. Durden F.M. and Elewsi, B. (1997). Fungal infections in HIV infected patients. Seminars in Cutaneous Medicine and Surgery. 16 (3): $200-212$.

[6]. Enwuru C.A., Ogunledun, A., Idika, N. Enwuru, N.N., Ogbonna F. and Aniedobe, M. (2008). Fluconaole Resisitant Opportunistic Oropharyngeal Candida and Non-albican Yeast-like Isolates from HIV Infected Patients Attending ARV Clinic In Lagos, Nigeria. African Health Science. 8 (3).

[7]. Kwamin F., Hewletts, S., Ndanu, T.A., Lartey, M. and Nartey, M.O. (2010). Incidence of Orophacial Lesions in Relation to CD4counts in HIV AIDS Patients at the Fevers 9 Unit-Korle-Bu Teaching Hospital. Ghana Dental J. 7: 22-26.

[8]. Liu, X., Liu, H., Guo, . and Luan W. (2006). Association of Asymptomatic Oral Candidal Carriage Oral Candidiasis and CD4 Lymphocyte Count in HIV-Positive Patients in China. Oral Diseases. 12 (1): $41-44$.

[9]. Maheshwari, M., Kaur R. and Chadha S. (2016). Candida specie Prevalence Profile in HIV Sero-positive Patients from a Major Tertiary Care Hospital in New Delhi India. Journal of Pthogen. 1-8.

[10]. Shahapur, P.R. and Bidri R.C. (2014). Recent trend in the spectrum of opportunistic infections in Human Immunodeficiency Virus Infected Individuals on Antiretroviral Therapy in South India. Journal of Natural Science, Biology and Medicine. 5 (2): 392 - 396.

[11]. Thanyasrisung, P., Kesakemol, P., Pipattanagovit, P., Yaungnak-Piboonratanakit, P., Pitiphat, W. and Matangkasombut O. (2014). Oral Candida carriage and Immune Status in Thai Human Immunodeficiency Virus-Infected Individuals. Journal of Medical Microbiology. $63: 753-759$.

[12]. UNAID report on the Global AIDS Epidemics 2013.

http://www.unaids.org/sites/default/files/en/media/unaids/contentassets/documents/epidemiology/2013/gr2013/UNAIDS.Global_re port_2013_en.pdf. 